\title{
A Study on Chromosomes in Hydra ${ }^{1}$
}

\author{
By \\ Hidejiro Niiyama \\ Zoological Institute, Faculty of Science, \\ Hokkaido Imperial University, Sapporo
}

(With 12 Textfigures)

Received November 19, 1943

Though a considerable number of studies have previously been contributed to the topic of the chromosomes of the Coelenterata, particularly on those of hydra by Dowing ('05, '08) and McConnell ('32, '36), and on fresh-water medusa by White ('30), these earlier works offer only fragmental knowledge involving quite insufficient data, and there have not been demonstrated as yet any correct evidences of chromosnmes in this group. A reinvestigation is thus desirable at present to establish exact accounts on the chromosomes of this group, in connection with its systematic relationship to the related groups of animals. On the other hand, the chromosome study of this group is greatly stimulated by the interesting sexual phenomena existing in this form. Here the present study was undertaken in this view in order to obtain accurate accounts of the chromosomes of hydra with three species as material obtainable in Hokkaido, directing special attention towards the number and morphological character of the chromosomes.

Here the author wishes to express his sincere gratitude to Prof. Kan Oguma and Assistant Prof. Sajiro Makino, under whose kind guidance the work was performed. The author is also greatly indepted to Dr. M. Kuwabara who kindly placed the material at the author's disposal.

\section{Material and Method}

The following three species of hydra, Hydra vulgaris attenuata Pall., Hydra circumcincta P. Sch. and Pelmatohydra oligactis (Pall.) furnished the material for the present study. They were all obtained in Hokkaido and have been cultured in this institute for several years under the special care of Dr. M. Kuwabara for the purpose of his experimental studies. The specimens were pipeted with culture medium, and put in a watch-glass. Then the water was absorbed by means of a piece of blottingpaper and the residue covered with the fixing solution. As fixative, weak Flemming's

1 Contribution No. 179 from the Zoological Institute, Faculty of Science, Hokkaido Imperial University, Sapporo. 
solution modified by the author for the study of the crustacean chromosomes was exclusively employed with satisfactory results. Through the usual paraffin method the sections were cut 5 micra in thickness, and stained with Heidenhain's iron-haematoxylin with a counterstain of lightgreen.

\section{Observations}

\section{Hydra vulgaris attenuata Pall.}

The present species is interesting in being a dioecious form. The experimental study made by Kuwabara ('36) shows that the sex is reversible in this species. The observations, therefore, were performed with the individuals in which the gonads were clearly apparent on the surface of body.

a) Observations on male individuals: Somatic divisions in ectodermal interstitial cells and also those in germ cells during spermatogenesis in the testis came under observation. Fig. 1 shows a polar view of the metaphase plate of an ectodermal interstitial cell. The chromosomes appear in the usual radial arrangement. After careful counting it was found that the number of chromosomes was invariably 32 . Though several other plates were examined, no fluctuation in number occurred in any case. Thus the diploid number of this species was determined as 32 . As is obvious in reference to the annexed figures (Figs. 1 and 9), the diploid complex consists of sixteen $\mathrm{V}$-shaped ones, two J-shaped ones, eight long rod-shaped ones, four short rod-shaped ones and two dot-like ones. In other words ${ }^{1}$. of 32 chromosomes sixteen elements are submesomitic,

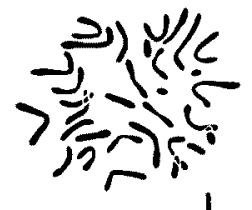

I

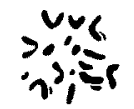

5

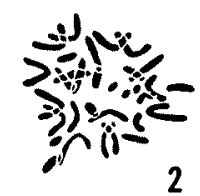

2

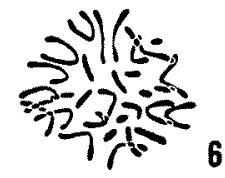

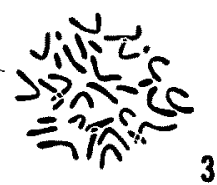

3

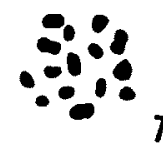

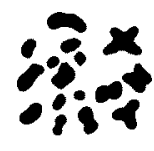

4

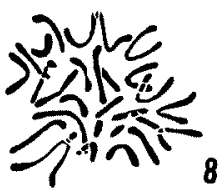

Figs. 1-8. 1-5 Chromosomes of Hydra vulgaris attenuata. $\times 3700 . \quad 1$, Ectodermal interstitial cell, 32 chromosomes. 2, Spermatogonium, 32 chromosomes. 3, Oogonium, 32 chromosomes. 4, Primary spermatocyte, 16 bivalents. 5, Secondary spermatocyte, 16 dyads. 6-7. Chromosomes of Hydra circumcincta. $\times 3700.6$, Ectodermal interstitial cell, 30 ehromosomes. 7, Primary spermatocyte, 15 bivalents. 8 . Chromosomes of Pellmatohydra oligactis. $\times 3700$. Ectodermal interstitial cell, 30 chromosomes.

1. For new terminology adopted here see Oguma ('42). 
two are subtelomitic and forteen are orthotelomitic. By comparison of their shape and size 32 elements are fairly sorted into 16 homologous pairs (Fig. 9).

The male gonad contains all kinds of germ cells from spermatogonia to spermatozoa showing active spermatogenesis. A good metaphase plate of the spermatogonial division is shown in Fig. 2. There are again found 32 chromoscmes in it, all of which are identical in their morphological characters to those already found in the interstitial cell.

The primary spermatocyte metaphase shows 16 tetrads constituting a beautiful equatorial plate (Fig. 4). Of these tetrads some are prominently large in size and assume a cross shape. It is certain that they are syntelomitic in structure and originate from the V-shaped elements in the diploid group. At anaphase of the first division, all the tetrads divide synchronously, neither precession nor succession were observed in any chromosomes.

The metaphase equatorial plate of the secondary spermatocyte shows 16 elements which consist of eight submesomitic, one subtelomitic and seven orthotelomitic elements, all being in dyad nature (Fig. 5). The complex shows a complete half set of the spermatogonial one.

b) Observations on female individuals: It is the author's regret that any suitable course of oogenesis could not be observed in the present material. For the material the specimens in which the ovaries were clearly observable were employed and the investigation was carried out with ectodermal interstitial cells. An example of the metaphase equatorial plate of an ectodermal interstitial cell is given in Fig. 3. The number of chromosomes was found to be 32 , being just the same as that of the male cell. The complement shows eight pairs of submesomitic elements, one pair of subtelomitic ones and seven pairs of orthotelomitic ones. The orthotelomitic elements are made up of four pairs of long-rods, two pairs of short-rods and one pair of dot-like elements (Fig. 10). Thus the female diploid complex is fairly identical in the morphological character of the component elements to the male complex. So far as the number of chromosomes and morphological character of the individual elements are concerned, there occurs no sexual difference of chromosomes in this species.

\section{Hydra circumcincta P. Sch.}

According to Kuwabara ('33), the present species is a monoecious form: the upper portion of the body is male and the lower part is female in one individual. Observations were made with interstitial cells of ectoderm and germ cells in the testis. The chromosome complex found in the interstitial cell is morphologically similar to that of the spermatogonial cell, as is the case with the previous species. Fig. 6 shows a metaphase equatorial plate of an ectodermal interstitial cell. The chromosome number 


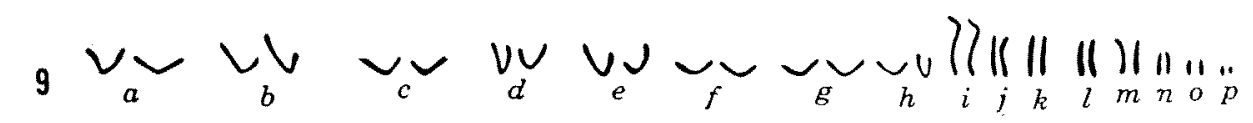

$10 \sim a$
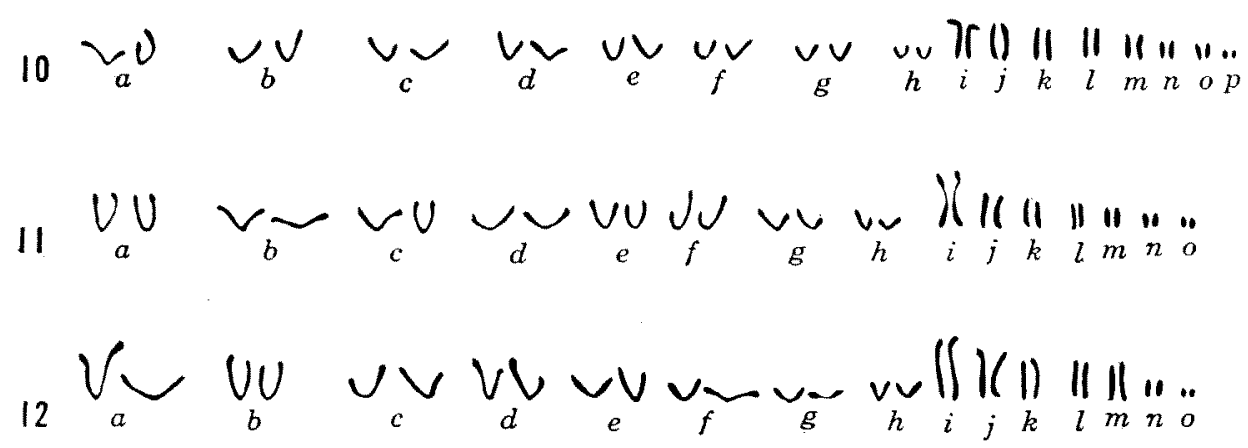

Figs. 9-12. Serial alignments of the paired chromosomes from the diploid complexes. $\times 3700$. 9, 10, Hydra vulgaris attenuata. 11, Hydra circumcincta. 12, Pelmatohydra oligactis.

(Fig. 9 corresponds to Fig. 1; Fig. 10 to Fig. 3; Fig. 11 to Fig. 6 and Fig. 12 to Fig. 8).

was observed to be 30 (as $2 n$ ), two less than that of the previous species. The diploid complex is composed of eight pairs of submesomitic chromosomes, one pair of subtelomitic ones and six pairs of orthotelomitic elements, in which latter four pairs are comparatively long, one pair is shortsized and the remaining one pair is extremely small (Fig. 11). The subtelomitic chromosomes show satellite-like structure, being composed of two parts connected by a fine thread (Fig. 11, i).

There are observable 15 bivalent elements in the metaphase plate of the primary spermatocyte (Fig. 7). All the bivalents are dumb-bell in shape. They divide synchronously into two equal halves at anaphase of the first division. The evidence thus shows that there is no indication for the occurrence of the particular chromosome, identical to the sexchromosome of the other forms of animals.

\section{Pelmatohydra oligactis (Pall.)}

The present species is reported by McConnell ('32, '36) as a dioecious form. But the material cultured by Dr. Kuwabara, on which the present study was based has repeated an asexual reproduction for several years and neither testis nor ovary was formed in any specimens. The observations, therefcre, were made only with the interstitial cell of ectoderm as the material. Fig. 8 is a representative of the metaphase plate of the interstitial cells. There are observable 30 chromosomes radially arranged. The diploid number thus determined is in complete coincidence with that of Hydra circumcincta. Of 30 elements, sixteen are submesomitic, two are subtelomitic and twelve are orthotelomitic. The orthotelomitic chromo- 
somes can be divided by their length into eight long-rods, two short-rods and two extremely small ones. They form 15 homologous pairs with equal shape and size (Fig. 12). No particular element could be observed.

\section{Remarks}

Referring to the literature, there are found several papers reporting the chromosomes of the Coelenterata published by previous authors, such as Dowing '05, '08; McConnell '32, '36; White '30. Dowing ('05, '08) investigated the chromosomes of Hydra dioecia, Hydra fusca and Hydra viridis, reporting that there were $12 \mathrm{~V}$-shaped chromosomes in the interstitial cell and also in the oogonial cell, while in the spermatogonial cell 12 elements of spherical ones were found. McConnell ('32, '36), working on Pelmatohyara oligactis found in America, reported that there were recognizable $12 \mathrm{~V}$-shaped chromosomes in mitosis of the interstitial cell. The number of chromosomes in these species is too low as compared with those established in the present study. Referring to the original papers of Dowing ('05, '08) and McConnell ('32, '36), it seems probable to the author that the results reported by Dowing and McConnell cannot be considered as valid. There is no doubt that the low number reported by them may be accounted for by the clumped condition of the chromosomes caused by faultry preservation.

Concerning the fresh-water medusa, Craspedacusta ryderi, White ('30) described 12 rod-shaped chromosomes to occur in anaphase of the first spermatocyte division. According to him, at metaphase of the primary spermatocyte division the chromosomes can not be clearly distinguished. Considered from his description and the figures given in his paper, the number of chromosomes reported for this form cannot be regarded as conclusive. It seems probable that the reported evidences are greatly affected by inadequate technique.

The dipioid numbers and chromosomal formula of the three species of hydra herein investigated are as follows:

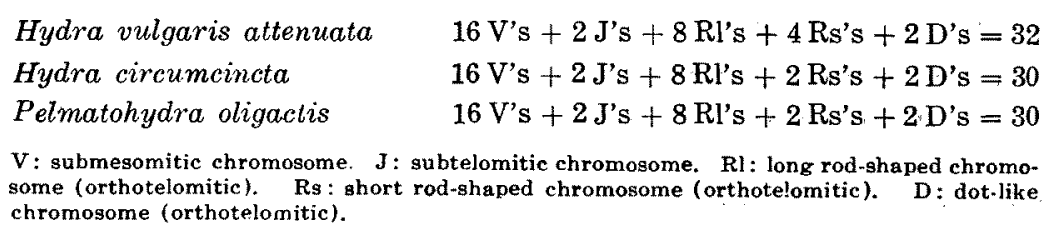

The above table indicates that in general the karyotypes of these three species nearly resemble each other. But in details there are some peculiar characteristics occurring for each species. Hydra vulgaris attenuato shows 32 chromosomes as diploid. This species is characterized by having two more minute chromosomes (Rs's) than in the other two species (Figs. 9. 
and 10). Hydra circumcincta is very prominent in carrying in its compliment a pair of satellite-like chromosomes of subtelomitic nature (Figs. 6 and 11), while in Pelmatohydra oligactis submesomitic chromosomes are comparatively larger than those in the other two species (compare Fig. 12 to Figs. 9 and 11 ).

In all species herein studied there was found no evidence for the presence of the particular chromosome to be considered as the sex-chromosome, neither from behaviour nor in structure.

\section{Summary}

The chromosomes of the following three species of hydra were investigated: Hydra vulgaris attenuata Pall., Hydra circumcincta P. Sch. and Pelmatohydra oligactis (Pall.)

The number of chromosomes in Hydra vulgaris attenuata was found to be 32 in diploid and 16 in haploid. The chromosomes show no sexual difference, so far as the morphological feature is concerned. Hydra circumcincta shows 30 chromosomes in diploid and 15 in haploid. The diploid number in Pelmatodydra oligactis was given as 30 .

The chromosomes were morphologically analized for every species and karyological comparison was made among them.

\section{Literature}

Dowing, E. R. 1905. The spermatogenesis of Hydra. Zool Jahrb. Abt f. Anat 21: 379426.

- 1908. The ovogenesis of Hydra. ibid. 28: 295-324.

Kuwabara, M. 1933. Two species of Hydra from Japan. Proc. Imp. Acad. 9: 643-645.

- 1936. Beiträge zur Kenntnis der Sexualität von Süßwasser-Hydroiden. I. Die sexuellen Rassen von Hydra attenuata. Jour. Fac. Sci. Hokkaido Imp. Univ. Ser. VI, Zool. 5: $95-112$.

McConnell, C. H. 1932. Zellteilungen bei Hydra. Mitosen in den epithelio-muskulären Zellen des Entoderms von Hydra. Zeits. mikr. Anat. Forsh. 28.

- 1936. Mitosis in Hydra. Mitosis in the indifferent interstitial cells of Hydra. Roux' Arch. Ent.-mech. Org. 135 : 202.

Oguma, K. 1942. Observations de formis compositionibusque chromosomatum et de dispositionibus eorum in tempore divisionis atque propositio aliquorum novorum terminorum. Jap. Jour. Gen. 18: 205-216.

White, W. E. 1930. Notes on a fresh-water medusa found in Stallworth Lake, Tuscaloosa, Alabama. Biol. Bull. 59 : 222. 